\title{
Suppression of Oscillatory Activity in Crustacean Pyloric Neurons: Implication of GABAergic Inputs
}

\author{
J. R. Cazalets, I. Cournil, M. Geffard, ${ }^{a}$ and M. Moulins \\ Laboratoire de Neurobiologie et Physiologie Comparées, CNRS and Université de Bordeaux I, F-33120 Arcachon, France
}

Generation of rhythmic pyloric motor output in the crustacean stomatogastric ganglion results from synaptic connections and cellular properties of a 14-cell network of pyloric neurons. These cellular properties are under the influences of modulatory inputs, which act, for the most part, in an activating mode, i.e., they enhance the bursting properties of the pyloric neurons and/or their ability to express their regenerative properties. Here we attempt to demonstrate that the pyloric motor output is also under the control of suppressive afferent inputs that are able to stop the pyloric rhythm in a long-lasting manner.

Immunohistochemistry, using GABA antibodies, indicates that GABAergic-like fibers are present in both the stomatogastric ganglion and its afferent nerve. Bath-applied GABA suppresses spontaneous pyloric rhythmic activity. This is due to an inability of the pyloric pacemakers to express their bursting properties. The suppressive effect of GABA is blocked by picrotoxin and mimicked by muscimol. Isolating the pyloric neurons from all descending spiking influences with tetrodotoxin demonstrates that exogenously applied GABA acts directly on the pyloric neurons. To confirm the existence of a physiological suppressive system for the pyloric motor pattern, we show that the stimulation of an afferent nerve, known to contain GABA-like fibers, also causes the cessation of rhythmic activity and the inability of the pyloric neurons to express their bursting properties.

It is increasingly evident that central rhythmic motor networks are under the control of modulatory inputs (Selverston and Moulins, 1985; Harris-Warrick, 1987). These inputs do not control the pattern of the output step by step, as can proprioceptive feedback, but have, instead, long-lasting instructive effects that shape the final motor output.

One of the best-known examples of such modulatory control is the 14- neuron network of the crustacean stomatogastric ganglion, which drives the alternating dilations and constrictions of the pyloric chamber (Moulins and Nagy, 1985; Marder, 1987). All the neurons involved in this network have the ability to

\footnotetext{
Received Nov. 17, 1986; revised Mar. 6, 1987; accepted Mar. 9, 1987.

We wish to thank E. Marder, S. L. Hooper, F. Nagy, and J. Simmers for critical comments on the manuscript and help in preparing the English version of the text. This work was supported by MRT Grant 85-C-1152.

Correspondence should be addressed to Jean-René Cazalets, Laboratoire de Neurobiologie et Physiologie Comparées, CNRS, Université de Bordeaux I, Place du Dr. Peyneau, F-33120 Arcachon, France.

a Present address: Laboratoire de Neuroimmunologie, Institut de Biologie Cellulaire et Neurochimie, CNRS, Domaine de Carreire, rue Camille Saint-Saens, F-33077, Bordeaux, France.

Copyright $C 1987$ Society for Neuroscience $0270-6474 / 87 / 092884-10 \$ 02.00 / 0$
}

produce bursting pacemaker potentials (BPPs; Miller, 1987), i.e., regenerative depolarizations and hyperpolarizations that underlie their bursting discharge. The voltage-dependent conductances responsible for these regenerative properties are under the control of modulatory inputs, since their removal decreases or turns off the production of pyloric BPPs, and hence rhythmic output (Moulins and Cournil, 1982; Nagy and Miller, 1987). Only a few modulatory input neurons to the pyloric network have been identified (Nagy et al., 1981; Russell and Hartline, 1981; Dickinson and Nagy, 1983; Nagy and Dickinson, 1983), but there is pharmacological evidence (Beltz et al., 1984; Claiborne and Selverston, 1984; Marder and Eisen, 1984; Marder, 1987) that suggests that this network is the target of a palette of such inputs. To date all the identified modulatory inputs (Russell and Hartline, 1981; Dickinson and Nagy, 1983; Nagy and Dickinson, 1983) and most of the putative neuromodulatory substances applied to the network have permissive effects on the pyloric output (Beltz et al., 1984; Hooper and Marder, 1984; Marder and Eisen, 1984; Harris-Warrick, 1987). They are all able to start the pyloric rhythm in a quiescent ganglion, or to enhance ongoing pyloric activity, even if in some cases there is a diminution in the discharge of some of the target neurons (Flamm and Harris-Warrick, 1986a, b).

The present study was undertaken to determine if this network also receives modulatory "suppressive inputs," that is, inputs that reduce and finally suppress its rhythmic activity. Marder and Paupardin-Tritsch (1978) have shown that iontophoretic application of GABA results in hyperpolarization and conductance increase in several pyloric neurons. We proceeded from this observation to determine if (1) the pyloric network receives GABAergic-like inputs, (2) exogenously applied GABA suppresses the ability of pyloric neurons to produce BPPs, and (3) some of the neuronal inputs to the pyloric network have suppressive effects on the pyloric output.

A brief report of this work has appeared elsewhere (Cazalets et al., 1985).

\section{Materials and Methods}

Experiments were performed on the European lobster Homarus gammarus and the cape lobster Jasus lalandii; although similar results were obtained from both species, all results reported here are from Homarus. The animals were kept in tanks of aerated and circulating seawater. Two experimental salines were used: artificial seawater (Moulins and Vedel, 1977), $400 \mathrm{~mm} \mathrm{NaCl}, 9.8 \mathrm{~mm} \mathrm{KCl}, 10.1 \mathrm{~mm} \mathrm{CaCl}_{2}, 52.6 \mathrm{~mm} \mathrm{MgCl}$, $27.8 \mathrm{mM} \mathrm{Na}_{2} \mathrm{SO}_{4}, 2.5 \mathrm{~mm} \mathrm{NaHCO}_{3}, 0.6 \mathrm{~mm} \mathrm{NaBr}$; and HEPES-buffered saline (Miller and Selverston, 1982), $479 \mathrm{~mm} \mathrm{NgCl}, 12.74 \mathrm{mM} \mathrm{KCl}$, $13.7 \mathrm{mM} \mathrm{CaCl}_{2}, 10 \mathrm{mM} \mathrm{MgSO}_{4}, 3.9 \mathrm{mM} \mathrm{Na}_{2} \mathrm{SO}_{4}, 5 \mathrm{~mm}$ HEPES. The $\mathrm{pH}$ of the salines was adjusted to 7.45 with $\mathrm{HCl}$ or $\mathrm{NaOH}$.

GABA immunohistochemistry. The anti-GABA antibodies used for immunohistochemistry were purified, by affinity chromatography, from 

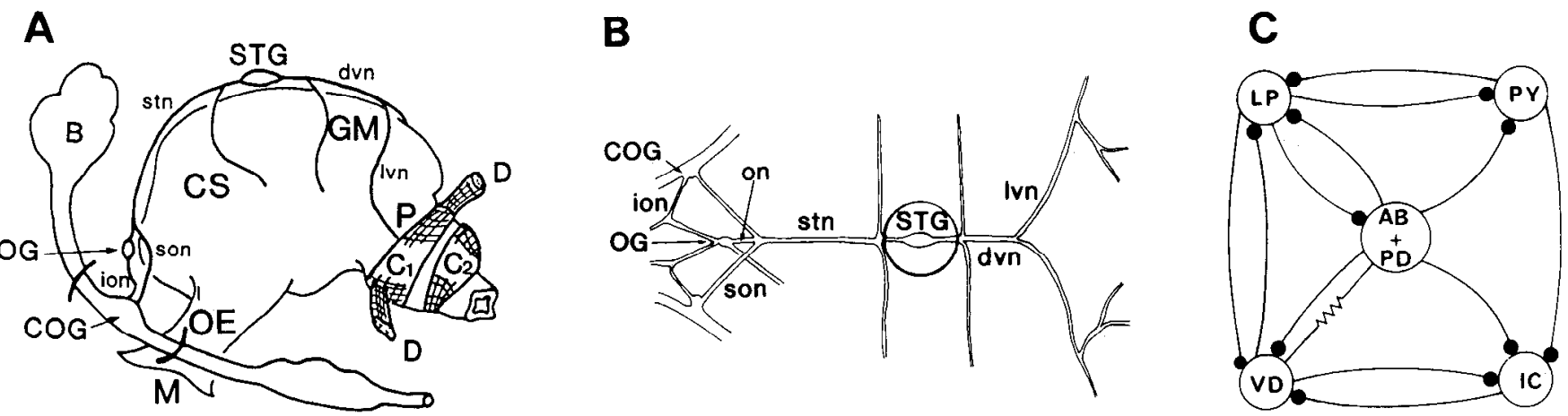

Figure 1. The stomatogastric nervous system of Homarus gammarus. A, Lateral view of the stomach showing the in situ location of nerves and ganglia on the dorsal part of the stomach and around the csophagus. B, The isolated nervous system. The circle around the STG represents the Vaseline pool through which drugs were superfused. $C$, Simplified wiring diagram of the pyloric motor network. $-\mathbf{-}$, Inhibitory synapse, - -n electrotonic junction. Abbreviations: $A B$, anterior burster interneuron; $B$, brain; $C_{1}$, early constrictor muscle; $C_{2}$, late constrictor muscle; $C O G$, commissural ganglion; $C S$, cardiac sac; $D$, dilator muscle; $d v n$, dorsal ventricular nerve; $G M$, gastric mill; $I C$, inferior cardiac motoneuron; ion, inferior esophageal nerve; $L P$, lateral pyloric motoneuron innervating $\mathrm{C}_{1} ; l v n$, lateral ventricular nerve; $M$, mouth; $O E$, esophagus; $O G$, esophageal ganglion; on, esophageal nerve; $P$, pylorus; $P D$, pyloric dilator motoneuron; $P Y$, pyloric motoneuron innervating $C 2$; son, superior esophageal nerve; $S T G$, stomatogastric ganglion; stn, stomatogastric nerve; $V D$, ventricular dilator motoneuron.

rabbit anti-GABA antiserum obtained by Seguela et al. (1984). The anti-GABA antiserum does not cross-react with glutamate, aspartate, or other related molecules, such as alanine, glycine, or taurine (Seguela et al., 1984).

The stomatogastric nervous system and the second and third abdominal ganglia were dissected in artificial seawater (adjusted to $\mathrm{pH} 6.2$ with $\mathrm{HCl}$ ) and fixed with $5 \%$ glutaraldehyde diluted in $0.4 \mathrm{M}$ cacodylate buffer (pH 7.75) for $90 \mathrm{~min}$. The nervous tissue was first reacted with $1 \%$ lysine for $1 \mathrm{hr}$, then with $0.1 \%$ sodium borohydrate for $1 \mathrm{hr}$ (to reduce the autofluorescence of the fixed tissue), and postwashed overnight at $4^{\circ} \mathrm{C}$ in PBS $(0.01 \mathrm{M}$ phosphate buffer containing $0.15 \mathrm{M} \mathrm{NaCl}), \mathrm{pH} 7.45$, to which was added $10 \%$ sucrose. Frozen sections $(18 \mu)$ were incubated for $18 \mathrm{hr}$ with the anti-GABA antibodies diluted $1 / 200$ in PBS containing $0.3 \%$ Triton X-100 and $10 \%$ BSA (in order to prevent nonspecific binding). After a $1 \mathrm{hr}$ wash in fresh PBS, the reaction of the anti-GABA antibodies was visualized by incubating the sections for $1 \mathrm{hr}$ with sheep anti-rabbit IgG linked to fluorescein isothiocyanate (FITC; Miles) diluted $1 / 100$ in PBS. After washing, the sections were mounted in a $1: 3$ mixture of PBS-glycerol and observed with a Leitz fluorescence microscope (excitation filter, 450-490 $\eta \mathrm{m}$; emission filter, $520 \eta \mathrm{m}$ ).

Absorption controls were carried out to test the specificity of antiGABA antibodies used in this procedure. The anti-GABA antibodies were diluted according to the normal procedure and preincubated for $24 \mathrm{hr}$ with or without the immunogen (GABA-glutaraldehyde-BSA). The 2 solutions were then centrifuged and the supernatants collected. Successive sections of the ganglia were treated alternately with the absorbed and nonabsorbed antibodies and subsequently observed and compared under fluorescence as above.

Electrophysiological techniques. After removing the stomatogastric nervous system from the dorsal part of the stomach (Fig. 1A), the isolated nervous system was pinned out in a Sylgard-lined petri dish (Fig. $1 B$ ) and maintained at $15^{\circ} \mathrm{C}$. Isolated preparations, corresponding to the "combined preparation" of Selverston et al. (1976) and consisting of the stomatogastric ganglion (STG), the paired commissural ganglia (COGs), and the esophageal ganglion (OG), were used. Intrasomatic recordings of pyloric neurons wcrc madc with glass microelectrodes filled with $2 \mathrm{M}$ potassium acetate $(r=15-20 \mathrm{M} \Omega)$. Neurons were identified as described for Homarus by Robertson and Moulins (1981) and for Jasus by Nagy (1981). For intracellular current injections, cell bodies were always penetrated with 2 microelectrodes, one for membrane potential recording and the other for current injection. Neuronal activity was recorded on a MP5521 Schlumberger tape recorder and visualized either on a 5113 Tektronix oscilloscope or a Gould ES 1000 electrostatic recorder. The effects of GABA and other drugs were studied by superfusing ( $1 \mathrm{ml} / \mathrm{min})$ the solutions through a Vaseline well $(2 \mathrm{ml} \mathrm{vol})$ built around the STG. GABA, picrotoxin (PTX), tetrodotoxin (TTX), and oxotremorine were obtained from Sigma; muscimol from Serva. Axonal conduction in nerves could be reversibly blocked by superfusion of an isotonic sucrose solution ( $750 \mathrm{~mm}$ ) (adjusted to $\mathrm{pH} 7.45$ with $\mathrm{NH}_{4} \mathrm{OH}$ ) through a Vaseline pool built around a desheathed portion of the appropriate nerve.

\section{Results}

\section{GABA-like immunoreactive projections to the $S T G$}

The lobster STG consists of a dense neuropil surrounded by neuronal somata. The axons of pyloric motoneurons project from the STG in several motor nerves, the principal one being the dorsal ventricular nerve (dvn) (Fig. 1B). The STG is connected to the anterior ganglia (COGs and $O G$ ) by a single nerve tract, the stomatogastric nerve (stn, Fig. $1, A, B$ ), which carries numerous input fibers to the STG. With anti-GABA antibodies we visualized immunoreactive fibers in the neuropil of the STG (Fig. 2, $A, D$ ), but none of the cell bodies in this ganglion were labeled. GABA-like immunoreactive fibers were also detected in the input nerve (stn, Fig. $2 B$ ), but not in the main output motor nerve (dvn, Fig. 2C). Although the results shown here are from IIomarus, identical results were obtaincd with Jasus. Since none of the cell bodies stained in the STG, it is likely that the immunoreactive neuropilar structures are the terminal processes of neurons whose somata are located in other ganglia and project to the STG via the stn. In Homarus, some of the immunoreactive fibers projecting to the STG probably originate in the $O G$, in which we saw at least 3 strongly immunoreactive cell bodies, and/or the COGs, which also contain some small immunoreactive cell bodies. The nerves that interconnect these ganglia (see Fig. $1 \mathrm{~A}$ ) also contain GABA-like immunoreactive fibers.

These results depend critically on the specificity of the antibodies. Although several tests have been carried out previously to establish this specificity (Seguela et al., 1984), we have performed 2 further controls. First, we demonstrated that the antibodies recognize identified GABAergic neurons in the lobster abdominal ganglion (Otsuka ct al., 1967). Figure $3 B$ shows that after treatment with the anti-GABA antibodies, the somata of these neurons, $I 2$, and $I 3$, were strongly immunoreactive. Several small-diameter cell bodies, probably including II of Otsuka et al. (1967), were also labeled, but numerous other neurons of the ganglion were not. Thus, the antibodies used in the present study do seem to recognize neuronal structures known to be 

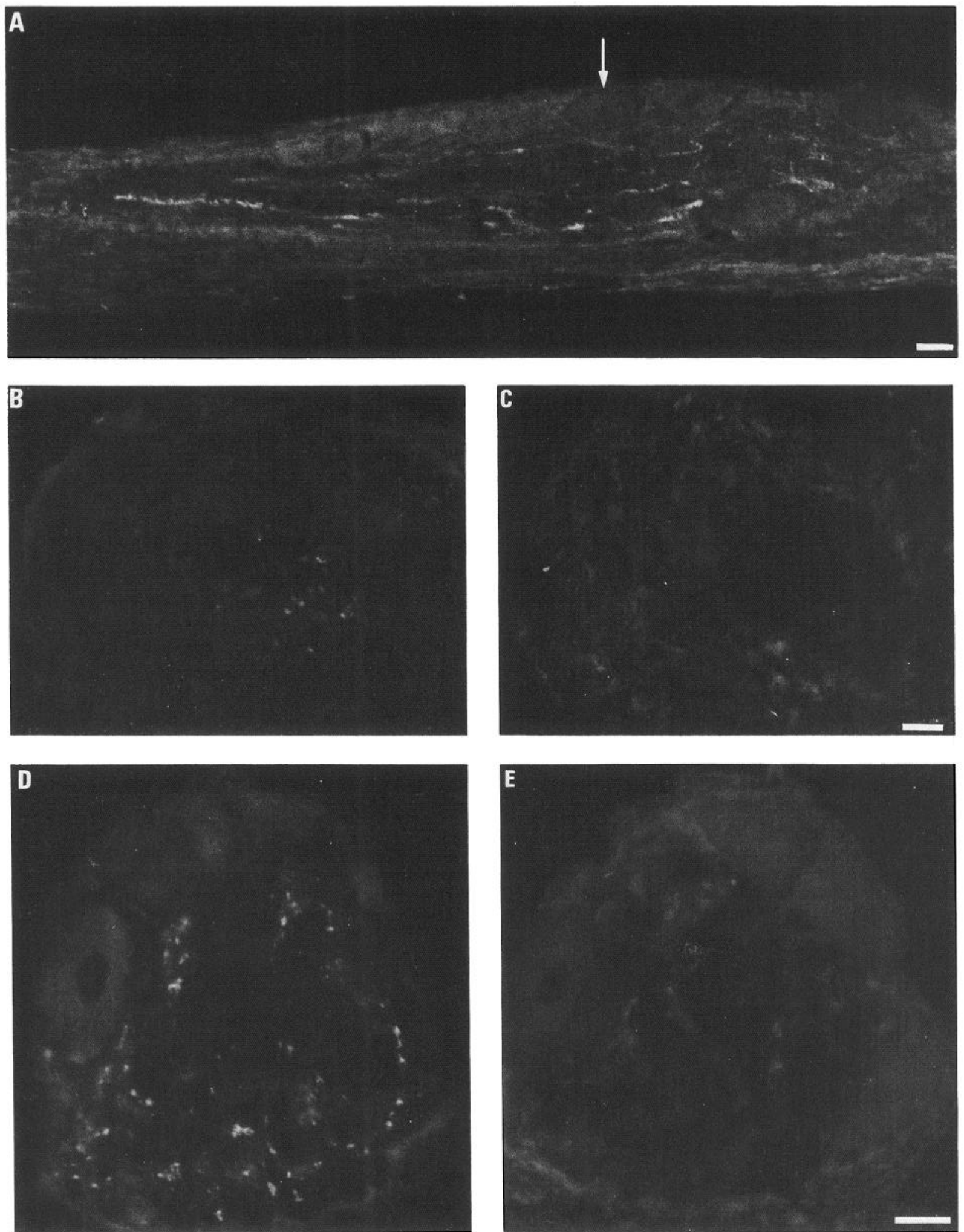

Figure 2. GABA-like immunoreactivity in and near the stomatogastric ganglion of Homarus. A, Longitudinal section of the stomatogastric ganglion after incubation with GABA antibodies (the stn is to the left, the dvn to the right). Note immunoreactive fibers in the neuropil and the absence of labeled cell bodies (arrow.) $B, C$, Immunoreactive fibers in a transverse section of the input nerve (stn; $B$ ) but not in a transverse section of the main motor nerve $(\operatorname{dvn} ; C) . D, E$, Absorption control on serial transverse sections of the stomatogastric ganglion: positive reaction observed in the neuropil of a section incubated with the nonabsorbed antibodies $(D)$ disappeared in the subsequent section when incubated with the absorbed antibodies $(E)$. Calibration lines; $50 \mu \mathrm{m}(A) ; 25 \mu \mathrm{m}(B-E)$. 

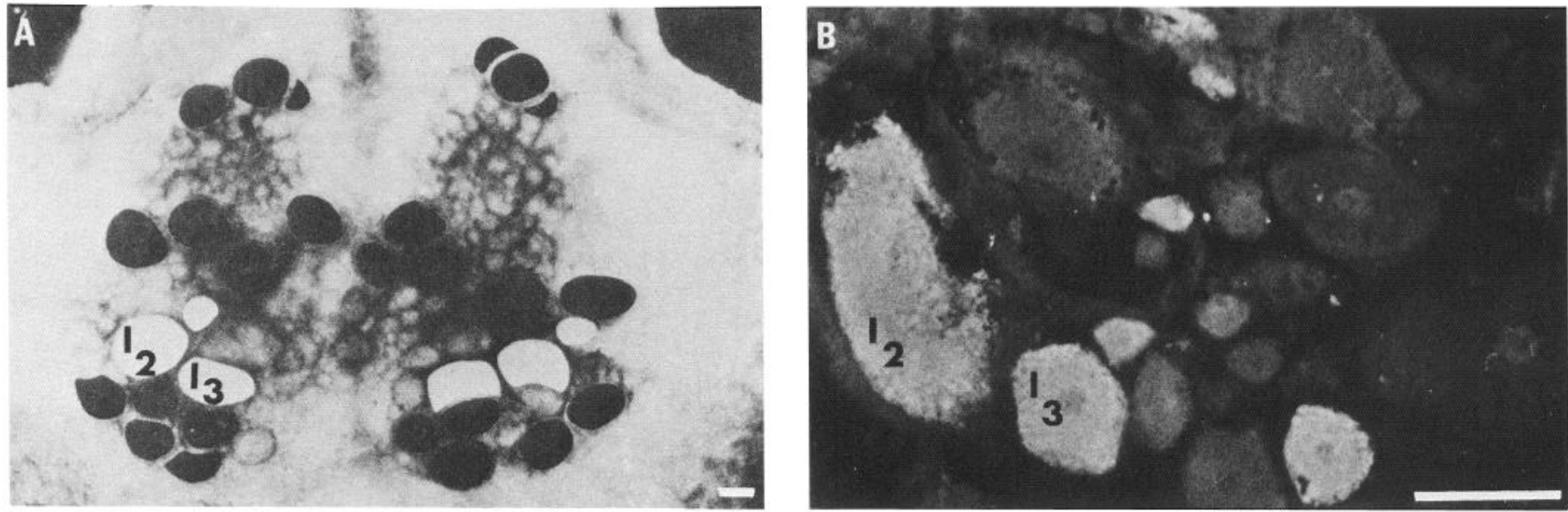

Figure 3. Cell bodies of known GABAergic inhibitory motoneurons in the abdominal ventral cord of the lobster Homarus labeled with GABA antibodies used in the present study. $A$, Map of the identified cells in the third abdominal ganglion. The cell bodies of inhibitory motoneurons are marked in white $(I 2, I 3)$ (from Otsuka et al., 1967). B, Longitudinal horizontal section of the third abdominal ganglion after antibody treatment; I 2 and probably I 3 neurons are immunoreactive. Calibration lines, $100 \mu \mathrm{m}$.

GABAergic. Second, after absorption by the antigen, the antibodies are unable to stain either identified GABAergic structures or the structures in the STG that were labeled prior to absorption. Absorption controls (see Materials and Methods) were performed on both the GABAergic neurons of the ventral nervous chain (data not shown) and the immunoreactive structures of the STG (Fig. 2, D, E). In all cases, the immunological reactions disappeared when the sections were treated with antibodies that had been incubated with the immunogen. Thus it seems that the antibodies specifically recognize GABA when coupled to proteins by glutaraldehyde, and we conclude that the structures labeled in the stn and STG contain GABA or at least a GABA-like compound.

\section{Suppression of the pyloric output by GABA application}

The activity of the pyloric neurons can be recorded intracellularly from in vitro preparations (Fig. $1 B$ ). The pyloric output consists of the sequential activity of 6 types of neurons (Fig.

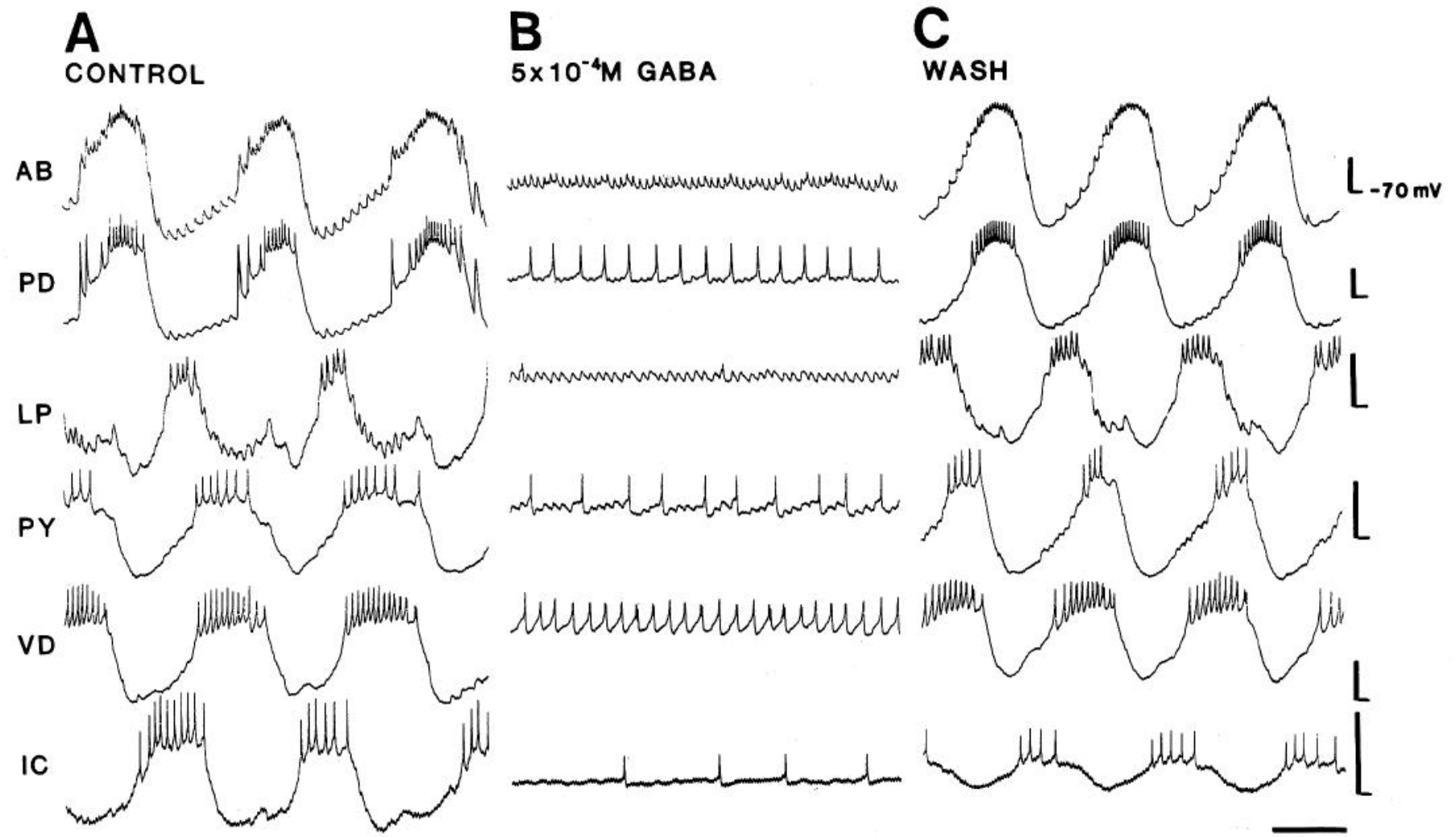

Figure 4. Bath-application of GABA on the STG causes cessation of pyloric rhythmic activity. Simultaneous intracellular recordings from the 6 types of pyloric cells (see Fig. 1C). $A$, Control. B, Superfusion over the STG of saline containing $5 \times 10^{-4} \mathrm{M}$ GABA stops the bursting discharges of the 6 neurons, although they continue to fire tonically at low frequency. $C$, Restoration of the rhythm 10 min after wash with normal saline. Horizontal bar, $0.5 \mathrm{sec}$; vertical bars, $5 \mathrm{mV}$. 

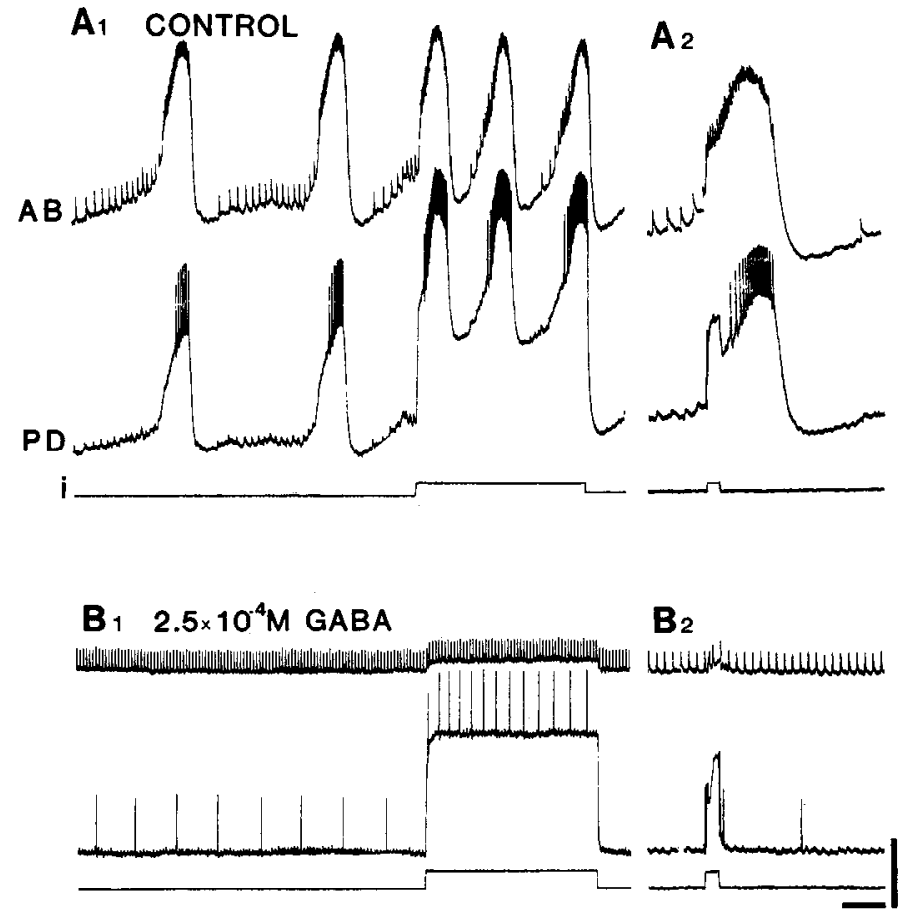

Figure 5. GABA applied to the STG causes the pyloric pacemaker neurons (PD and $\mathrm{AB}$ ) to be unable to fire rhythmic bursts of action potentials. $A$, Control. Before GABA application, the 2 pacemakers were oscillating spontaneously. Sustained depolarization (by current injection into PD) increased the frequency of oscillation $\left(A_{t}\right)$, while a brief depolarization provoked an immediate, full oscillation $\left(A_{2}\right)$. B , During GABA application $\left(2.5 \times 10^{-4} \mathrm{M}\right)$, a long-duration depolarization did not induce any oscillations $\left(B_{l}\right)$ and a brief depolarization was unable to provoke a regenerative response $\left(B_{2}\right)$. Horizontal bars, $1.25 \mathrm{sec}\left(A_{1}\right.$, $\left.B_{1}\right) ; 0.5 \mathrm{sec}\left(A_{2}, B_{2}\right)$. Vertical bars, $12 \mathrm{mV}$ (PD); $15 \mathrm{mV}$ (AB); and 30 $\mathrm{nA}$ for injected current $(i)$.

$4 A$ ). The 2 pyloric dilator motoneurons (PD) and the anterior burster interneuron $(\mathrm{AB})$ are electrically coupled, have endogenous oscillatory properties, and act as pacemakers for the pyloric network (Miller, 1987). They rhythmically inhibit all the other pyloric neurons, the synaptic interactions of which (Fig. $1 C$ ) determine the timing of their bursts of action potentials and hence the structure of the pattern. The motor pattern recorded from isolated stomatogastric nervous systems is comparable to that recorded by electromyography in the intact animal (Rezer and Moulins, 1983). In most isolated preparations, the pyloric neurons discharge rhythmically for hours at frequencies varying from 1 to $0.2 \mathrm{~Hz}$, depending on the preparation. In preparations in which the pyloric neurons oscillate at $1 \mathrm{~Hz}$ (Fig. $4 A$ ), complete cessation of all rhythmic activity was obtained by superfusing the STG with saline containing $5 \times$ $10^{-4}$ M GABA (Fig. $4 B$ ). With preparations in which the spontaneous pyloric rhythm was initially slower, the same result was obtained using $10^{-4} \mathrm{M}$ GABA. The effects of GABA application are rapidly reversible, oscillatory pyloric activity being completely restored after $10 \mathrm{~min}$ of washing with normal saline (Fig. 4C). In an earlier study, Marder and Paupardin-Tritsch (1978) found that GABA causes hyperpolarization and a conductance increase in pyloric neurons. Moreover, the membrane conductances that underlie the slow-wave oscillations are voltage-dependent and can be inactivated by steady hyperpolarization (Russell and Hartline, 1982). However, the following observa- tions indicate that the cessation of oscillations caused by GABA is not due simply to a hyperpolarizing effect.

\section{Suppression of the oscillatory properties of the pyloric pacemakers by $G A B A$ application}

The generation of pyloric motor activity depends largely on endogenous neuronal properties (Russell and Hartline, 1978) that are expressed only under the influence of modulatory inputs arising from"anterior centers" (Miller and Selverston, 1982; Moulins and Cournil, 1982; Russell and Hartline, 1982). These properties can be tested by (1) sustained artificial depolarization, which evokes oscillatory behavior in a nonoscillating neuron (see Fig. 9A), or an increase in the frequency of an already oscillating neuron (Fig. $5 A, 1$ ); or by (2) a brief depolarization, which triggers a full regenerative depolarizing response that outlasts the activating depolarizing pulse (Fig. $5 A, 2$ ). These tests were performed on the pyloric pacemaker neurons in the presence of GABA. Sustained depolarization (even at high current levels) did not evoke cycling, but only increased the tonic firing of the stimulated neurons (Fig. $5 B, 1$ ), while brief, strong depolarizations failed completely to trigger oscillatory potentials (Fig. 5B,2).

The following results further reinforce the notion that, in the presence of GABA, the pyloric neurons are no longer able to express their bursting propertics. Extraccllular stimulation of the stn is known to activate input fibers that promote the bursting potential of pyloric neurons; in a a system where the pyloric neurons are not cycling, a brief stimulation of the stomatogastric nerve can initiate and sustain the oscillatory activity of the pyloric neurons (Fig. $6 A$; note the long-term activating effect of this stimulation). In the presence of $2.5 \times 10^{-4} \mathrm{M}$ GABA, however, a longer stimulation at the same frequency had no such activating effect on the bursting potential of the $P D$ and $A B$ neurons (Fig. 6B). Poststimulus injection of depolarizing current, which would be expected to counteract a simple GABAmediated membrane hyperpolarization, was also ineffective in triggering oscillations.

These experiments thus show that the inability of the pyloric neurons to burst in the presence of GABA is not due only to a simple membrane hyperpolarization; rather, GABA must cause either the inactivation of a conductance directly involved in BPP production or an increase in a steady hyperpolarizing conductance that shunts those currents that generate bursts.

\section{Pharmacological characterization of the $G A B A$ effect}

To eliminate the possiblity of a nonspecific action of GABA on the pyloric neurons, and to begin characterization of the membrane receptors involved in its suppressive effect, several antagonists and agonists of GABA were tested. PTX is known to block the action of GABA in many preparations (Takeuchi, 1976). In a STG in which the PD neurons were cycling strongly and spontaneously (Fig. $7 \mathrm{~A}$ ), the application of saline containing $3.5 \times 10^{-4} \mathrm{M}$ GABA silenced the PD neuron (Fig. $7 B$ ). However, saline containing the same concentration of GABA applied in the presence of PTX was without effect on PD bursting activity (Fig. 7C), as was bath-applied PTX alone (Fig. 7D). Bicucculine, a well-known GABA antagonist (Curtis et al., 1970), was effective only at high concentrations $\left(10^{-3} \mathrm{M}\right)$, and its effect was less reproducible than that of PTX. This is consistent with the results of Marder and Paupardin-Tritsch (1978), who found that $10^{-4}$ M PTX partially suppressed the hyperpolarizing response of 

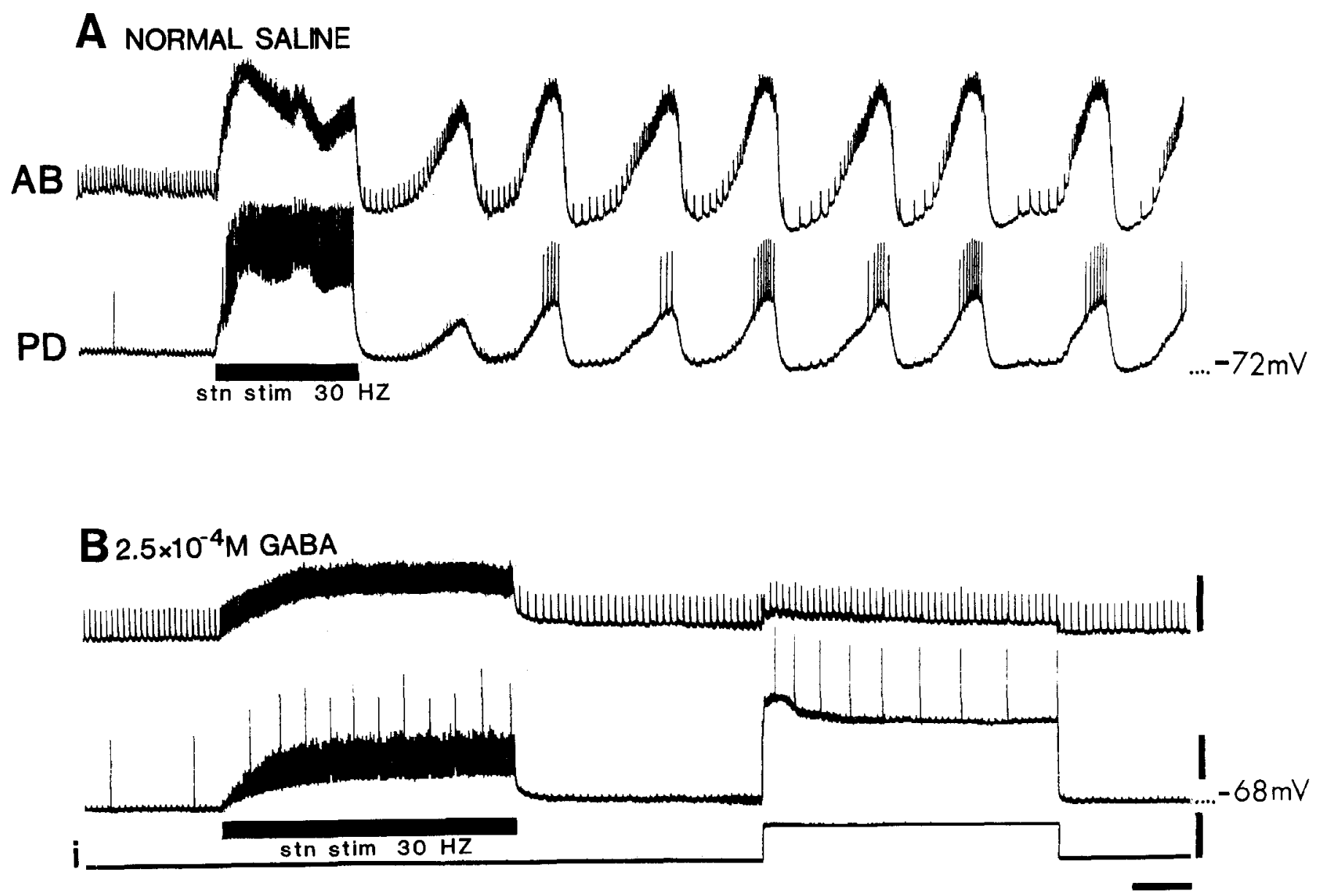

Figure 6. In the presence of GABA, the bursting properties of the pacemaker $(A B, P D)$ neurons can no longer be unmasked by stimulation of the stn. $A$, In a preparation that was not cycling, stn stimulation induced long-term oscillatory activity in the penetrated neurons. $B$, During superfusion of the ganglion with $2.5 \times 10^{-4} \mathrm{M} \mathrm{GABA}$, stronger stimulation (stim) of the stn was unable to induce neuronal oscillations, and a sustained depolarization of PD produced only tonic firing. Horizontal bar, $1 \mathrm{sec}$; vertical bars, $10 \mathrm{mV}, 10 \mathrm{nA}$.

STG neurons to iontophoretically applied GABA and that bicucculine $\left(10^{-4} \mathrm{M}\right)$ had no effect.

Muscimol, a potent agonist of GABA (Krogsgaard-Larsen and Arnt, 1979), had actions similar to those of GABA, but was effective at lower concentrations. Bath-applied muscimol $(7.5 \times$ $\left.10^{-6} \mathrm{M}\right)$ reduced the rhythmic activity of an oscillating PD neuron (cf. Fig. 7, E, F). At higher muscimol concentrations $\left(5.5 \times 10^{-5} \mathrm{M}\right)$, the oscillatory activity of the PD neuron stopped completely (Fig. $7 G$ ). As for GABA, this effect was reversible; a few minutes after the beginning of washing with normal saline, the PD oscillations were completely restored (Fig. $7 H$ ).

\section{The suppressive effects of GABA are TTX-resistant}

All the above results were obtained from preparations in which the STG remained connected to the premotor centers (Fig. 1B). It has previously been shown that the pyloric neurons receive extrinsic neuromodulatory inputs that have an activating effect on their oscillatory behavior. Thus it remains possible that the inactivating effects of GABA seen here may be due to presynaptic inhibition (Kirk and Wine, 1984) of these activating inputs. To test this possibility, the pyloric neurons were isolated from all spiking synaptic inputs by superfusing the STG with saline containing $2 \times 10^{-7} \mathrm{M}$ TTX. Under these conditions, the pyloric pacemakers stopped oscillating (Fig. $8 B$ ), but it was pos- sible to reinduce nonspiking pyloric oscillatory behavior by bathapplication of a muscarinic agonist, oxotremorine (Fig. $8 \mathrm{C}$ ), which mimics the activating effects of an identified cholinergic input (Nagy et al., 1985). The critical experimental observation was that, although completely deafferented, oxotremorine-activated pacemaker neurons were still sensitive to GABA. Again, bath-application of saline containing $10^{-4} \mathrm{M}$ GABA caused complete cessation of oscillatory activity (Fig. $8 D$ ). At this GABA concentration, a brief current pulse injected into a PD neuron still triggered a reduced oscillation in the PD neuron, as well as in the electrically coupled $\mathrm{AB}$ interneuron. A single (reduced) oscillation was also obtained by steady depolarization of the PD neuron (Fig. 8D). However, with a slightly increased GABA concentration $\left(2.5 \times 10^{-4} \mathrm{M}\right)$, the pacemaker neurons completely lost their regenerative ability: Even large-amplitude current pulses or strong, sustained depolarizations failed to trigger oscillations (Fig. $8 E$ ). After several minutes of wash with saline containing TTX and oxotremorine alone, the cycling activity of these neurons was restored (Fig. $8 F$ ). These results show that, for oxotremorine-induced BPPs in the presence of TTX, the suppressive effects of GABA do not occur via mechanisms presynaptic to the target neurons. We have no reason to believe that spontaneous BPPs are qualitatively different from BPPs induced by oxotremorine, and thus it is likely that, in normal 
A

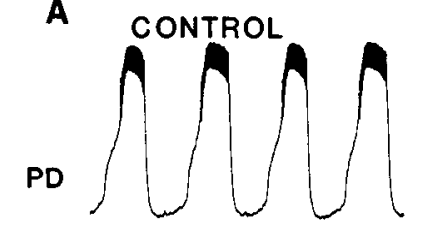

B $3.5 \times 10^{-4} \mathrm{M}$ GABA
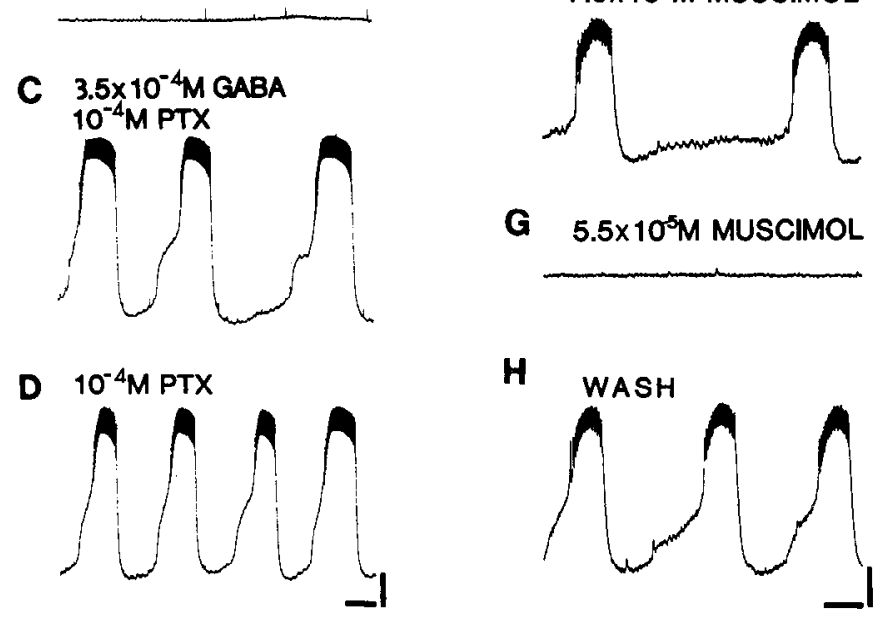

Figure 7. The action of GABA is antagonized by PTX and mimicked by muscimol. $A-D$, The oscillatory behavior of a PD neuron $(A)$ was suppressed by bath-application of GABA containing saline $(B)$. However, the same concentration of GABA was ineffective in the presence of PTX $(C)$. Bath-application of PTX alone did not modify the cycling of the PD neuron $(D) . E-H$, Bath-applied muscimol diminished $(F)$ or suppressed $(G)$ the oscillatory activity of a PD neuron (compare to $E$ ). This effect was reversible; $8 \mathrm{~min}$ after the beginning of the wash with normal saline, the oscillatory activity was completely restored $(H)$. Horizontal bars, $0.5 \mathrm{sec}(A-D)$; $1 \mathrm{sec}(E-H)$. Vertical bars, $10 \mathrm{mV}$.

saline as well, GABA's suppressive effects are due to direct action on the pyloric pacemaker neurons.

Comparable suppressive effects can be obtained by stimulating STG input fibers

To demonstrate that the pyloric pacemaker neurons receive inactivating GABAergic inputs requires selective stimulation of identified GABAergic fibers and evidence that these inputs have a suppressive effect on the oscillatory behavior of the pyloric pacemakers. To date, such input neurons have not been identified. However, it has been possible to show that electrical stimulation of multifiber input nerve to the STG can cause the cessation of pyloric rhythmic activity in a way similar to that of bath-applied GABA. Stimulation of the stn itself cannot be used because this input nerve contains many activating fibers and its stimulation normally initiates or enhances the oscillatory activity of the pyloric neurons (see Fig. $5 A$ ). We therefore used the esophageal nerve (on), which we know by immunohistochemistry contains GABA-like fibers (see above), but which, unlike the stn, carrics relativcly few activating elements that descend to the STG. In the experiment shown in Figure $9 A$, we recorded from a PD neuron that was quiescent, probaby owing to hyperpolarization, since sustained depolarization could evoke oscillation at a constant frequency (Fig. 9, upper trace). However, a similar sustained depolarization applied $2 \mathrm{sec}$ after a 25 $\mathrm{Hz}, 10 \mathrm{sec}$ stimulation of the on failed to produce any oscillatory activity (second trace, Fig. 9A). Thus, following stimulation of the on, the PD neuron was unable to express its oscillatory properties. This is directly comparable to the experiment shown in Figure $9 B$, in which the oscillatory behavior of a PD neuron is suppressed by on stimulation. This experiment also shows that on stimulation produces not only poststimulus hyperpolarization of PD, but also the loss of its oscillatory properties; a sustained depolarization immediately after the stimulation is totally unable to produce oscillations.

Since the on clearly contains fibers that have direct excitatory effects on the PD neuron (as can be seen during on stimulation in Fig. 9), the subsequent inactivation of the PD neuron could be due to post-tetanic depression rather than to the stimulation of suppressive fibers. Although this possiblity cannot be completely ruled out, PD neurons never exhibit long-term posttetanic depression in response to high-frequency firing induced either by depolarizing current injection or by stimulation of known excitatory synaptic inputs. Moreover, the fact that strong inactivation of the PD ncuron also follows a brief, low-frequency stimulation of the on (data not shown) is difficult to reconcile with an effect resulting from post-tetanic depression.

These 2 experiments also show that on stimulation has longlasting suppressive effects on the PD neuron. In the experiment shown in Figure $9 A$, the ability to produce BPPs was not completely recovered until $45 \mathrm{sec}$ after on stimulation, while in that shown in Figure $9 B$, although BPPs could be obtained by depolarization some $35 \mathrm{sec}$ after stimulation, recovery of spontaneous oscillatory activity took as long as $2 \mathrm{~min}$. Again, it is difficult to explain such long-term inactivation in terms of posttetanic depression; rather, the time course of these effects suggests inactivation via stimulation of modulatory suppressive fibers. Finally, it is important to note that the PD neuron inactivation seen in Figure $9 B$ was obtained from a preparation in which the COGs were removed. This suggests that these effects were the result of the activation of a direct pathway that originated in or passed via the OG en route to the STG.

\section{Discussion}

The present study shows that the single input nerve (stn) to the stomatogastric ganglion in at least 2 decapod crustaceans contains GABA-like immunoreactive fibers that ramify in the STG. Moreover, bath-applied GABA suppresses the ongoing rhythmic activity of the pyloric network located in the STG and this appears to be due to a direct action of GABA on pyloric neurons. Consistent with these observations is that similar inactivating effects can be obtained by direct electrical stimulation of the on which contains GABA-like fibers and runs into the stn. These results, therefore, suggest that, in addition to the modulatory activating inputs to the pyloric neurons that are responsible for their ability to produce BPPs (Marder, 1987), there exist modulatory inactivating inputs that are able to suppress the ability of pyloric neurons to produce BPPs. An important role in this suppression appears to be played by neurons containing GABA.

\section{GABAergic neurons in the crustacean stomatogastric nervous system}

The immunohistochemical results presented here were obtained with antibodies whose selectivity had been tested previously (Seguela et al., 1984). Moreover we have shown that these antibodies recognize identified neurons in the Homarus ventral nerve cord known to be GABAergic (Otsuka et al., 1967). These 
A

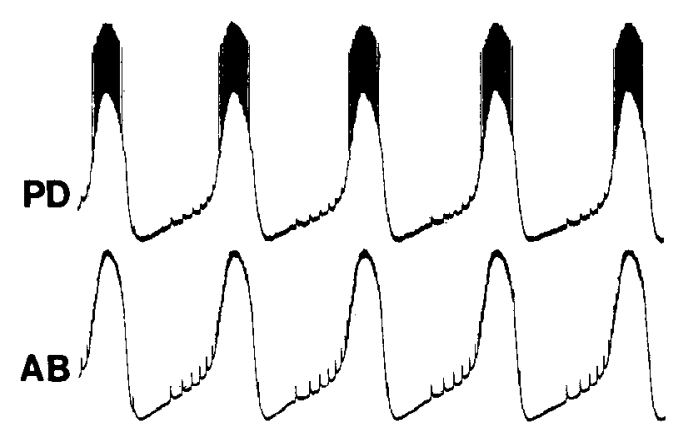

B

$2 \times 10^{-7} M$ TTX

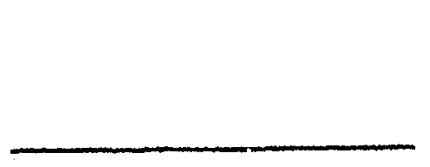

C

\section{$2 \times 10^{-7} \mathrm{M}$ TTX $+10^{-5} \mathrm{M}$ OXOTREMORINE}

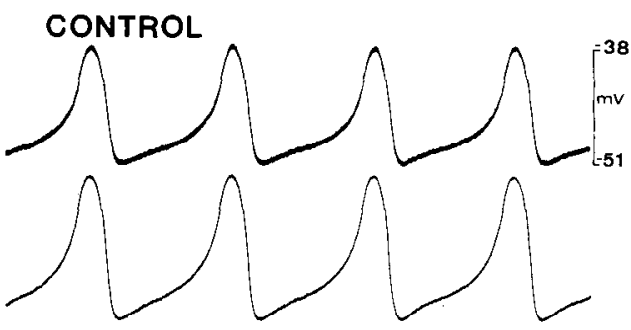

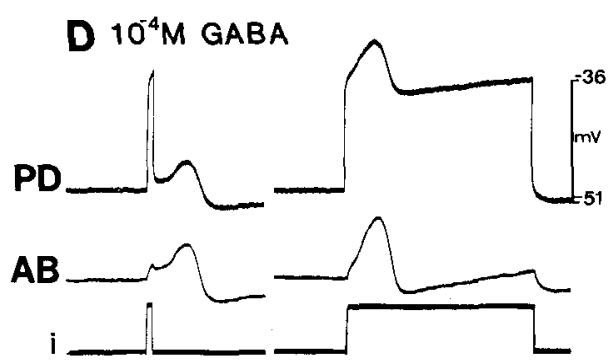

E $2.5 \times 10^{-4} \mathrm{M}$ GABA
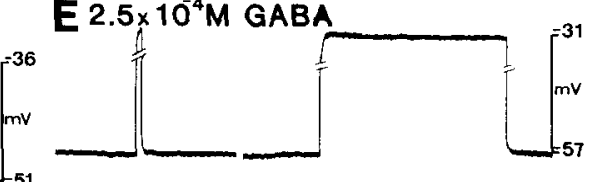

F WASH

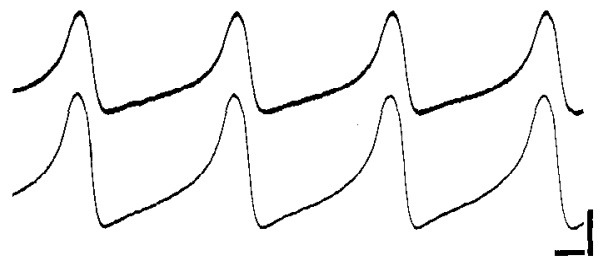

Figure 8. GABA suppresses pharmacologically induced oscillatory properties of "isolated" pyloric pacemaker cells. $A$, In normal saline the AB and PD neurons oscillated with a burst of spikes on the crest of each oscillation. $B$, Superfusion on the STG of saline containing $2 \times 10^{-7} \mathrm{M}$ TTX blocked spiking in the PD and $\mathrm{AB}$ neurons, which also stopped cycling. $C$, Cycling (without spikes) was then induced by the addition of $10^{-5} \mathrm{M}$ oxotremorine in the saline. $D$. Subsequent addition of $10^{-4} \mathrm{M}$ GABA gradually suppressed the oscillatory properties of the neurons. However, with this GABA concentration, an oscillation could still be triggered either by a short depolarizing pulse or with a prolonged depolarization. $E$, After application of GABA at a higher concentration $\left(2.5 \times 10^{-4} \mathrm{M}\right)$, injection of depolarizing current completely failed to trigger oscillations. $F$, Washing the preparation with saline containing TTX and oxotremorine alone reversed the effects of GABA. Horizontal bar, $1 \mathrm{sec}$; vertical bars, $10 \mathrm{mV}$ (AB neuron) and $3 \mathrm{nA}$.

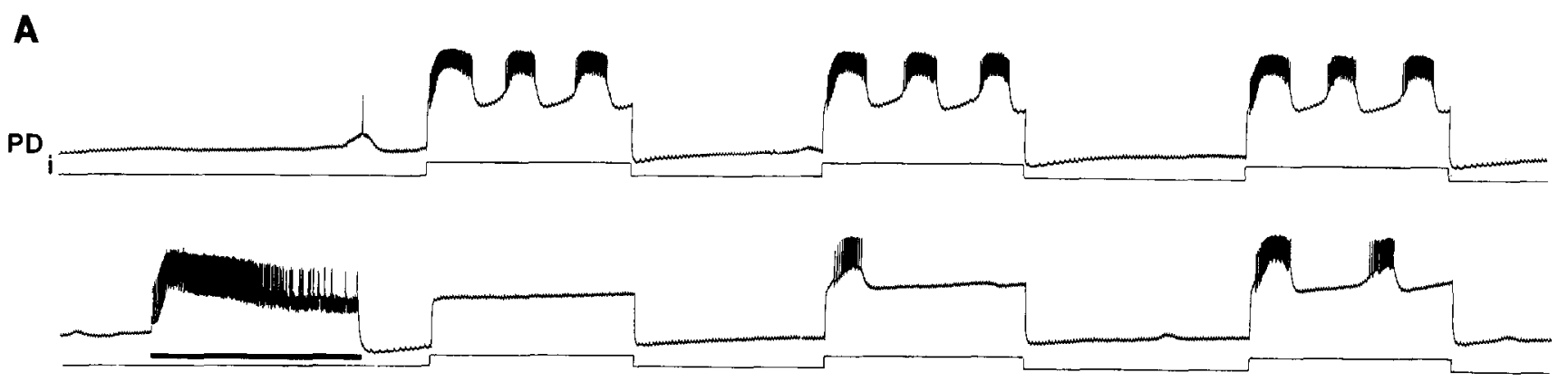

B

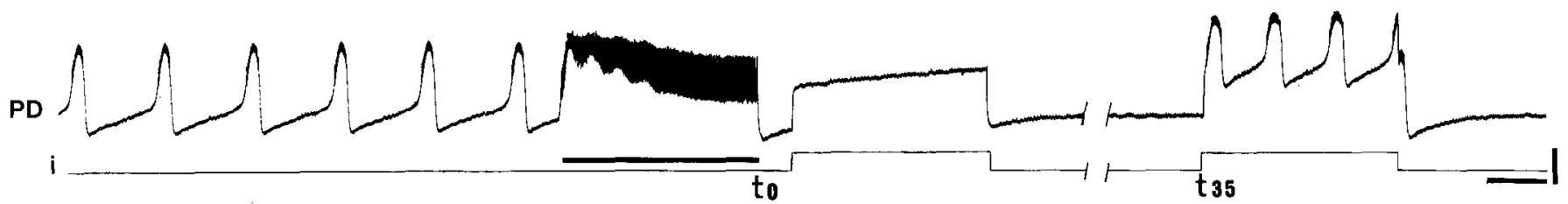

Figure 9. Direct stimulation of STG input fibers can suppress the oscillatory properties of the pyloric pacemaker PD neuron. $A$, Sustained depolarizations $(10 \mathrm{sec})$ induced by current injection $(i)$ into the cell body of a quiescent PD neuron evoked oscillations at a constant frequency (upper recording). After a $10 \mathrm{sec}$ stimulation of the esophageal nerve at $25 \mathrm{~Hz}$ (horizontal bar, second recording), the same depolarization of the PD neuron was unable to induce oscillations. The expression of oscillatory properties of the PD neuron returned slowly, but recovery was still not complete $14 \mathrm{sec}$ after the end of the stimulation (third depolarization). B, Similar stimulation of the on (horizontal bar) suppressed the oscillatory behavior of a previously active PD neuron. This effect was long-lasting, as subsequent sustained depolarization of PD did not evoke oscillations until $35 \mathrm{sec}(t 35)$ after the on stimulation $(t 0)$. Note, in $B$, that the COGs had heen removed and the PD neuron oscillations were induced by superfusion on the STG of saline containing $5 \times 10^{-6} \mathrm{M}$ oxotremorine. Horizontal bar, $1.75 \mathrm{sec}$; vertical bar, $15 \mathrm{mV}$. 
immunohistochemical results are consistent with biochemical analyses that show that glutamic acid decarboxylase (GAD) activity can be detected in those regions of the stomatogastric nervous system where immunoreactive structures have been identified (I. Cournil, unpublished observations). It is our conclusion, therefore, that GABAergic neurons are present in this nervous system, and that they probably originate in the $O G$ and/or COGs and project to the $\mathrm{SIG}$ via the stn.

By contrast, none of the cell bodies in the STG and none of their axons in the STG output ncrve cxhibit any immunorcactivity. This negative result is important for two reasons. First, it is well established that all chemical synapses within the pyloric network in the STG are inhibitory (Selverston et al., 1976). Several of these synapses, where the $A B$, lateral pyloric (LP), pyloric (PY), and inferior cardiac (IC) neurons are presynaptic (for details, see Fig. 1), are blocked by the GABA antagonist PTX (Bidaut, 1980; Eisen and Marder, 1982), and for this reason they were thought to be possibly GABAergic. However, Eisen and Marder (1982) have shown that the IPSPs evoked centrally in the pyloric network by these neurons are similar, in terms of reversal potential and ionic dependence, to glutamate responses, which are also blocked by PTX (Marder and Paupardin-Tritsch, 1978). In addition, most of these neurons are motoneurons and there is strong evidence that at their neuromuscular junctions they release glutamate (Lingle, 1980). Thus, their conclusion was that these neurons used glutamate at both their central and peripheral synapses. Our results are consistent with this conclusion, since none of the STG neurons exhibited GABA-like immunoreactivity.

Second, it was important, in the present study, to know whether any synaptic connections made by pyloric neurons within the STG were mediated by GABA. Our rationale for using exogenously applied GABA was to mimic the effects of putative GABAergic inputs extrinsic to the pyloric network. This approach would not have been possible if GABA was implicated in the synaptic relationships within the network.

\section{Inactivation of pyloric rhythmicity}

Numerous studies on the pyloric network have reaffirmed the notion that modulatory control is exerted by superior centers on the pyloric network. Russell (1979) initially observed that sucrose blockade of the unique afferent nerve (stn) to the STG produced a considerable decrease in activity of the pyloric neurons. Subsequent reports (Miller and Selverston, 1982; Moulins and Cournil, 1982; Nagy and Miller, 1987) have shown that suppression of afferent extrinsic inputs to the network caused the loss of the regenerative properties of the pyloric neurons, i.e., their ability to produce regenerative bursts of action potentials. Moreover, bath-application experiments have established that numerous putative neuromodulators, such as acetylcholine, dopamine, serotonin, octopamine, and peptides (proctolin, FMRFamide, substance $P$ ), have an activating effect on the pyloric neural network (for a review, see Marder, 1987). Parallel with this, an identified cholinergic neuronal input (Dickinson and Nagy, 1983; Nagy and Dickinson, 1983) that is able to induce or enhance, in a long-term manner, the BPP ability of the pyloric neurons has been described. Thus, all evidence to date has shown that the higher centers exert activating control of pyloric neurons.

However, in vivo, the pyloric muscles do not always operate at the relatively high and regular cycle frequencies observed in vitro (Rezer and Moulins, 1983), but instead exhibit activities with a wide range of low and irregular frequencies. It is possible that, in the intact animal, diminished pyloric frequency could be due to a corresponding decrease in the activating modulatory inputs. Here we suggest that a decrease in pyloric rhythmicity may be due not only to a reduction of activating systems, but to the existence of an inactivating system operating antagonistically. A strong argument for the latter is the presence in the stomatogastric nervous system of fibers whose stimulation leads to a loss of bursting properties in the pyloric pacemaker neurons (Fig. 9).

A strong candidate as mediator of this suppressive role is GABA. Our data show that GABA can exert a suppressive action on the BPP production of pyloric neurons (Figs. 5, 6), and that this effect is direct (Fig. 8). Moreover, the existence of GABA-like fibers in the stn, as determined by immunohistofluorescence (Fig. 2), reinforces the idea of a suppressive role for this molecule on pyloric neurons of the stomatogastric ganglion.

Although we do not yet know whether GABA's effect on the pyloric pacemaker neurons is due to "attacks" on conductances directly involved in the generation of BPPs or whether it causes a shunt by conductance increase, it is clear that, functionally, the pyloric pacemakers lose their bursting ability in its presence. We also do not yet know if the inactivation of oscillations in the other pyloric neurons is due to a direct action of $\mathrm{G} A \mathrm{~B} \Lambda$ or is the result, in part at least, of the loss of the drive by the PD$\mathrm{AB}$ neuron pacemaker group.

One possible problem stems from the relatively high GABA concentrations needed to obtain a complete cessation of the pyloric rhythm. We have found that, depending on the preparation, although GABA begins to act at $10^{-4} \mathrm{M}$, a concentration of $5 \times 10^{4} \mathrm{M}$ is generally required to stop cycling. Even though a dose-response relation (frequency of cycling versus GABA concentration) is seen (data not shown), the range of concentration over which this can be observed is too small to establish a dose-response curve. It is possible that the bath-application technique used in our experiments could be responsible for the apparently high concentrations of GABA needed. GABA applied with this technique on the well-studied GABA neuromuscular junction of the crayfish starts to induce conductance increases only at $10^{-5} \mathrm{M}$, and maximal effects are reached at $5 \times 10^{-4} \mathrm{M}$ (Takeuchi, 1976). As has been proposed by other authors (Deisz et al., 1984), the existence in crustaceans of a highly efficient glial uptake system for GABA could limit the access of GABA to postsynaptic receptors when it is bath-applied. The observation that muscimol, a poor substrate for uptake, acts at concentrations 10 times lower than GABA supports this idea.

The role of such an inactivating system in the overall control of the pyloric network could be multiple. First, it could operate in a dynamic equilibrium with permissive influences, so as to finely adjust the membrane properties and level of activity of the pyloric neurons. Second, by acting in a more drastic manner, this suppressive system could cause cessation of pyloric activity, as happens during "nonfeeding" periods in the intact animal (Rezer and Moulins, 1983). A third role of such inactivation, and one we have observed occurring spontaneously in some in vitro preparations (unpublished data), could be to "liberate" certain pyloric neurons from the pyloric network to allow participation in other rhythmic activities (for example, cardiac activity; Moulins and Vedel, 1977). 
In conclusion, suppressive control of the pyloric network of the lobster can be exerted by modifying the ability of the pyloric pacemakers to produce BPPs. Experiments are now in progress to show if this control extends as well to the pyloric constrictor neurons. We also intend to define the conductances implicated in this GABAergic suppression of BPP ability and to characterize the afferent inputs responsible for this inactivation of the pyloric output.

\section{References}

Beltz, B., J. S. Eisen, R. Flamm, R. M. Harris-Warrick, S. L. Hooper, and E. Marder (1984) Serotonergic innervation and modulation of the stomatogastric ganglion of three decapod crustaceans (Panulirus interruptus, Homarus americanus and Cancer irroratus). J. Exp. Biol. 109: 35-54.

Bidaut, M. (1980) Pharmacological dissection of pyloric network of the lobster stomatogastric ganglion using picrotoxin. J. Neurophysiol. 44: $1089-1101$.

Cazalets, J. R., I. Cournil, and M. Moulins (1985) GABAergic inactivation of burst generating oscillations in lobster pyloric neurons. Soc. Neurosci. Abstr. 11: 1022.

Claiborne, B. J., and A. I. Selverston (1984) Histamine as a neurotransmitter in the stomatogastric nervous system of the spiny lobster. J. Neurosci. 4: 708-721.

Curtis, D. R., A. W. Duggan, D. Felix, and G. A. Johnston (1970) GABA, bicucculine and central nervous inhibition. Nature 226: $1222-$ 1224.

Deisz, R. A., M. Dose, and H. D. Lux (1984) The time course of GABA action on the crayfish stretch receptor: Evidence for a saturable GABA uptake. Neurosci. Lett. 47: 245-520.

Dickinson, P. S., and F. Nagy (1983) Cuntrol of a central pattern generator by an identified modulatory interneurone in crustacea. II. Induction and modification of plateau properties in pyloric neurons. J. Exp. Biol. 105: 59-82.

Eisen, J. S., and E. Marder (1982) Mechanisms underlying pattern generation in lobster stomatogastric ganglion as determined by selective inactivation of identified neurons. III. Synaptic connections of electrically coupled pyloric neurons. J. Neurophysiol. 48: 1392-1415.

Flamm, R. E., and R. M. Harris-Warrick (1986a) Aminergic modulation in lobster stomatogastric ganglion. I. Effects on motor pattern and activity of neurons within the pyloric circuit. J. Neurophysiol. 55: 847-865.

Flamm, R. E., and R. M. Harris-Warrick (1986b) Aminergic modulation in lobster stomatogastric ganglion. II. Targets neurons of dopamine, octopamine, and serotonin within the pyloric circuit. J. Neurophysiol. 55: 866-881.

Harris-Warrick, R. M. (1987) Chemical modulation of central pattern generators. In Neural Control of Rhythmic Movements, A. H. Cohen, S. Rossignol, and S. Grillner, eds., Wiley, New York (in press).

Hooper, S. L., and E. Marder (1984) Modulation of a central generator by two neuropeptides, proctolin and FMRFamide. Brain Res. 305: $186-191$.

Kirk, M. D., and J. J. Wine (1984) Identified interneurons produce both primary afferent depolarization and presynaptic inhibition. Science 225: 854-856.

Krogsgaard-Larsen, P., and J. Arnt (1979) GAB $\Lambda$ receptor agonists: Relationship between structure and biological activity in vivo and in vitro. In GABA Biochemistry and CNS Functions, P. Mandel and $\mathrm{F}$. V. de Feudis, eds., pp. 303-321, Plenum, New York, London.

Lingle, C. (1980) The sensitivity of decapod foregut muscles to acetylcholine and glutamate. J. Comp. Physiol. 138: 187-199.

Marder, E. (1987) Neurotransmitters and neuromodulators. In The Crustacean Stomatogastric System: A Model for the Study of Central Nervous Systems, A. I. Selverston and M. Moulins, eds., pp. 263306, Springer-Verlag, Berlin, Heidelberg, New York.

Marder, E., and J. S. Eisen (1984) Electrically coupled pacemaker neurons respond differently to the same physiological inputs and neurotransmitters. J. Neurophysiol. 51: 1362-1374.
Marder, E., and D. Paupardin-Tritsch (1978) The pharmacological properties of some crustacean neuronal acetylcholine, $\gamma$-aminobutyric acid and L-glutamate responses. J. Physiol. (Lond.) 280: 213-236.

Miller, J. P. (1987) Pyloric mechanisms. In The Crustacean Stomatogastric System: A Model for the Study of Central Nervous Systems, A. I. Selverston and M. Moulins, eds., pp. 109-136, Springer-Verlag, Berlin, Heidelberg, New York.

Miller, J. P., and A. I. Selverston (1982) Mechanisms underlying pattern generation in lobster stomatogastric ganglion as determined by selective inactivation of identified neurons. II. Oscillatory properties of pyloric neurons. J. Neurophysiol. 48: 1378-1391.

Moulins, M., and I. Cournil (1982) All-or-none control of the bursting properties of the pacemaker neurons of the lobster pyloric pattern generator. J. Neurobiol. 13: 447-458.

Moulins, M., and F. Nagy (1985) Extrinsic inputs and flexibility in the motor output of the lobster pyloric neural network. In Model Neural Networks and Behavior, A. I. Selverston, ed., pp. 49-67, Plenum, New York, London.

Moulins, M., and J. P. Vedel (1977) Programmation centrale de l'activité motrice rythmique du tube digestif antérieur chez les crustacés décapodes. J. Physiol. (Paris) 73: 471-510.

Nagy, F. (1981) Etude de l'expression d'activités motrices rythmiques organisées par des générateurs paucineuroniques du système nerveux stomatogastrique des crustacées décapodes. Doctoral thesis, Université de Bordeaux I.

Nagy, F., and P. S. Dickinson (1983) Control of a central pattern generator by an identified modulatory interneurone in crustacea. I. Modulation of the pyloric motor output. J. Exp. Biol. 105: 35-58.

Nagy, F., and J. P.Miller (1987) Pyloric pattern generation in Panulirus interruptus is terminated by blockade of activity through the stomatogastric nerve. In The Crustacean Stomatogastric System: A Model for the Study of Central Nervous Systems, A. I. Selverston and M. Moulins, eds., pp.136-139, Springer-Verlag, Berlin, Heidelberg, New York.

Nagy, F., P. S. Dickinson, and M. Moulins (1981) Modulatory effects of a single neuron on the activity of the pyloric pattern generator in crustacea. Neurosci. Lett. 23: 167-173.

Nagy, F., J. A. Benson, and M. Moulins (1985) Cholinergic inputs reduce a steady outward $\mathrm{K}^{+}$current allowing activation of a $\mathrm{Ca}^{++}$ conductance which underlies the burst-generating oscillations in lobster pyloric neurons. Soc. Neurosci. Abstr. 11: 1022.

Otsuka, M., E. A. Kravitz, and D. D. Potter (1967) Physiological and chemical architecture of a lobster ganglion with particular reference to gamma-amino-butyrate and glutamate. J. Neurophysiol. 30: 725752 .

Rezer, E., and M. Moulins (1983) Expression of the crustacean pyloric pattern generator in the intact animal. J. Comp. Physiol. 153: 17-28.

Robertson, R. M., and M. Moulins (1981) Oscillatory command input to the motor pattern generators of the crustacean stomatogastric ganglion. I. The pyloric rhythm. J. Comp. Physiol. 143: 453-463.

Russell, D. F. (1979) CNS control of pattern generators in the lobster stomatogastric ganglion. Brain Res. 177: 598-602.

Russell, D. F., and D. K. Hartline (1978) Bursting neural networks: A reexamination. Science 200: 453-456.

Russell, D. F., and D. K. Hartline (1981) A multiaction synapse evoking both EPSPs and enhancement of endogenous bursting. Brain Res. 223: $19-38$

Russell, D. F., and D. K. Hartline (1982) Slow active potentials and bursting motor patterns in pyloric network of the lobster, Panulirus interruptus. J. Neurophysiol. 48: 914-937.

Seguela, P., M. Geffard, R. M. Buijs, and M. Le Moal (1984) Antibodies against $\gamma$-aminobutyric acid: Specificity studies and immunocytochemical results. Proc. Natl. Acad. Sci. USA 81: 3888-3892.

Selverston, A. I., and M. Moulins (1985) Oscillatory neural network. Annu. Rev. Physiol. 47: 29-48.

Selverston, A. I., D. F. Russell, J. P. Miller, and D. G. King (1976) The stomatogastric nervous system: Structure and function of a small neural network. Prog. Neurobiol. 7: 215-290.

Takeuchi, A. (1976) Studies of inhibitory effects of GABA in invertebrate nervous systems. In GABA in Nervous System Function, E. Roberts, T. N. Chase, and B. Tower, eds., pp. 255-267, Raven, New York. 